\title{
Editorial: The future of ionic liquids in the field of nanomaterials for energy applications
}

Rachel M. Frazier PhD

Research Engineer, Alabama Innovation and Mentoring of Entrepreneurs, The University of Alabama, Tuscaloosa, Alabama, USA

\section{Scott K. Spear PhD}

Research Engineer, Alabama Innovation and Mentoring of Entrepreneurs, The University of Alabama, Tuscaloosa, Alabama, USA
The field of nanomaterials is expansive and there are many technological applications of nanomaterials, some of which converge with commercial markets that benefit society. In particular, the use of nanomaterials in energy applications is growing and includes cross-cutting fields such as solar, energy storage, and greenhouse gas capture and sequestration. Some of the more popular nanomaterials used in energy applications include metal oxides and carbonbased materials. There have been significant research advances in nanomaterials for energy applications; however, transitioning these lab findings to industry still needs attention. The adoption of nanomaterials in the energy industry has been relatively slow and one critical need is occupational and environmental precautions during industrial handling and processing of nanomaterials. Another need is a system that allows easy incorporation of nanomaterials into existing industrial processes. These are areas of opportunity in which ionic liquids have distinct advantages. This themed issue on ionic liquids introduces the role these organic materials play in nanomaterials for energy with the goal of demonstrating how ionic liquids can meet existing needs within the field of nanomaterials and the field of energy. In addition, this issue aims to generate concepts centered on the possibilities ionic liquids have to offer in the emerging intersection of nanomaterials for energy.

Ionic liquids are organic salts with attributes that distinguish them from other organic materials. Their unique physical and chemical properties make them valuable and versatile materials. Unlike commodity chemicals, such as conventional organic solvents, ionic liquids are specialty chemicals and are slowly and steadily emerging in the marketplace. The precise dollar amount of this emerging market is difficult to obtain, however, a recent report values the global market for ionic liquids at US\$300M in 2010 and projects it to be worth US $\$ 3.4 \mathrm{~B}$ by $2020 .{ }^{1}$ The commercial applications of ionic liquids are far reaching and the markets associated with these applications are large and growing (see Table 1).

Due in part to the versatility of these materials, ionic liquids have received an amazing amount of interest over the past 15 years. As Prof. Endres points out in his recent editorial on the interfaces of ionic liquids, ${ }^{2}$ ionic liquids have experienced an unprecedented worldwide interest culminating in 4000 papers being published in 2011 on this class of liquid salts. There is much literature on the application of ionic liquids to nanomaterial fabrication, solar cells, biomass processing, and $\mathrm{CO}_{2}$ capture. A quick look using the SciFinder ${ }^{\circledR}$ search engine gives the recent rise of interest in the intersection of ILs and nano. A search of keywords "ionic liquid nano" comes back with 3112 journal articles with $99 \%$ of those articles appearing within the last 10 years. In 2002, there were only 19 papers published in this field; however, in 2011 there were 700 papers published. Further refinement of the search terms to "ionic liquid nano" and "energy" yields 96 journal articles, all appearing within the last 10 years. These "ionic liquid nano energy" articles present a similar trend toward rising interest with the first article appearing in 2003 and increasing to 25 in 2011. And, with numbers already out for 2012, there is no indication that the role ionic liquids will play in nanomaterials and energy will decline anytime soon. On the contrary, 2012 articles are set to outpace 2011 numbers.

So exactly how do ionic liquids fit into the intersection of nanomaterials and energy? Ionic liquids can be engineered to meet specific needs of a given field and they offer the opportunity to tailor nanomaterials for applications in energy. Ionic liquids offer nanomaterials processing advantages including size and shape control during nanomaterial synthesis. ${ }^{3}$ Another attribute of ionic liquids is that they provide a medium to control dust generation without nanoparticle agglomeration. In fact, companies are already offering products that combine the advantages of ionic liquids without sacrificing the inherent value of nanomaterials. Iolitec, $\mathrm{GmbH}$, and Iolitec, Inc. are leading providers of nanomaterials in ionic liquids for customers in supercapacitors, nanoparticle synthesis, and solar cells. See http://www.iolitec.com or http://www.iolitec-usa.com for further details. Forming and packaging nanomaterials in ionic liquids provides benefits to industrial processing for example, allowing easy incorporation of nanoparticles into composites. This offers a convenient way to get nanomaterials into existing processes in the energy industry, impacting the supply chain.

The range of ionic liquids is extensive and the required properties vary with the end use. What is appropriate for electrolytes 


\begin{tabular}{llcl} 
IL commercial application & Market & Market size (USD) & Year \\
Battery electrolyte & Portable battery powered products & $\$ 423 \mathrm{~B}$ & 2010 \\
& & $\$ 611 \mathrm{~B}$ & 2016 \\
Alternative solvent to volatile organic & Alternative chemical products and processing & $\$ 194 \mathrm{~B}$ & 2007 \\
compounds & & $\$ 420 \mathrm{~B}$ & 2013 \\
Solvents for $\mathrm{CO}_{2}$ capture in flue gas & Carbon capture and storage technologies & $\$ 89 \mathrm{~B}$ & 2007 \\
& & $\$ 236 \mathrm{~B}$ & 2012 \\
Dye sensitized solar cell electrolyte & Advanced materials and devices for renewable & $\$ 18 \mathrm{~B}$ & 2010 \\
& energy & $\$ 32 \mathrm{~B}$ & 2016 \\
Solvents in biogas scrubbers & Biogas upgrading & $\$ 0 \cdot 05 \mathrm{~B}$ & 2010 \\
& & $\$ 0 \cdot 34 \mathrm{~B}$ & 2016 \\
\hline Source: Market values compiled from reports available on bccresearch.com. &
\end{tabular}

Table 1. The commercial applications of ionic liquids and the value of those markets.

may not be suitable for $\mathrm{CO}_{2}$ capture. Additional, and sometimes peripheral, aspects to consider are the environmental benefits ionic liquids have to offer. ${ }^{4}$ Imidazolium-based ionic liquids represent a widely studied class of these materials, and many papers within this themed issue cover recent findings regarding this type of ionic liquid in the crossover between nanomaterials and energy. ${ }^{3,5,6}$ In addition, this issue presents derivatives and alternatives for designing new ionic liquids suitable for nanomaterials and energy. ${ }^{4,7}$ Perhaps the greatest advantage of using ionic liquids is the flexibility in design afforded by the vast choice of ions.

From an introduction and brief history of ionic liquids ${ }^{4}$ to the latest applications of using ionic liquids to process nanomaterials for energy applications, ${ }^{3,5-7}$ the Ionic Liquids and Nanomaterials for Energy themed issue covers the role ionic liquids will play in emerging nanotechnology and energy areas and the cross-over between the two fields. The future of ionic liquids in the field of nanomaterials for energy applications is promising and this special issue highlights some of the potential.

\section{REFERENCES}

1. Helmut Kaiser Consultancy. Ionic liquids 2030: Ionic liquid technologies, markets, applications, companies and developments worldwide 2008 to 2020 and 2030, 2010, http://www.hkc22.com/ ionicliquids.html

2. Endres, F. Interfaces of Ionic Liquids. Phys Chem Chem Phys. 2012, 14(15): 5008-5009.

3. Singh, T.; Trivedi, T. J. and Kumar, A. Ionic liquid-assisted preparation of $\mathrm{ZnO}$ nanostructures. Nanomaterials and Energy. 2012, 1(3): 207-215.

4. Dilip, M. Cradle to grave: How green are ionic liquids? Nanomaterials and Energy. 2012, 1(3): 193-206

5. Jamikorn, S.; Arunchaiya, M.; Friedrich, D. and Dittrich, T. Solar cell based on PEO, inorganic cations, and ionic liquids. Nanomaterials and Energy. 2012, 1(3): 216-224

6. Maxim, M. L.; Sun, N.; Wang, H.; Sterner, J. R.; Haque, A. and Rogers R. D. Reinforced magnetic cellulose fiber from ionic liquid solution. Nanomaterials and Energy. 2012, 1(3): 225-236

7. Bara, J. E. and Shannon, M. S. Beyond 1,3-difunctionalized imidazolium cations. Nanomaterials and Energy. 2012, 1(3): 237-242 